\title{
A Generalized Measure for the Optimal Portfolio Selection Problem and its Explicit Solution
}

\author{
Zinoviy Landsman ${ }^{1, *}$, Udi Makov ${ }^{1}$ and Tomer Shushi ${ }^{1,2}$ \\ 1 Actuarial Research Center, Department of Statistics, University of Haifa, Mount Carmel, 3498838 Haifa, \\ Israel; udimakov@gmail.com (U.M.); tomershushi@gmail.com (T.S.) \\ 2 Department of Economics and Business Management, Ariel University, Ariel 40700, Israel \\ * Correspondence: landsman@stat.haifa.ac.il
}

Received: 4 January 2018; Accepted: 26 February 2018; Published: 6 March 2018

\begin{abstract}
In this paper, we offer a novel class of utility functions applied to optimal portfolio selection. This class incorporates as special cases important measures such as the mean-variance, Sharpe ratio, mean-standard deviation and others. We provide an explicit solution to the problem of optimal portfolio selection based on this class. Furthermore, we show that each measure in this class generally reduces to the efficient frontier that coincides or belongs to the classical mean-variance efficient frontier. In addition, a condition is provided for the existence of the a one-to-one correspondence between the parameter of this class of utility functions and the trade-off parameter $\lambda$ in the mean-variance utility function. This correspondence essentially provides insight into the choice of this parameter. We illustrate our results by taking a portfolio of stocks from National Association of Securities Dealers Automated Quotation (NASDAQ).
\end{abstract}

Keywords: global optimization; fractional programming; linear constraints; mean-variance model; optimal portfolio selection; Sharpe ratio

\section{Introduction}

The portfolio selection problem is of both theoretical and of practical interest (Castellano and Cerqueti 2014; Li and Hoi 2014; Shen et al. 2014; Fletcher 2015; Fulga 2016; Ray and Jenamani 2016). The pioneering work of Markowitz (1952) (see Elton and Gruber. 1987; Steinbach 2001) on optimal portfolio selection (OPS) introduced the classical mean variance (MV) approach

$$
M V(R)=E(R)-\lambda \operatorname{Var}(R), \lambda>0,
$$

which has been studied extensively in the financial literature ( Landsman and Valdez 2003; Li and Hoi 2014; Bera et al. 2015; Graham et al. 2015; Qin 2015; Markowitz 2014). Here $R=\pi^{T} \mathbf{X}$ is the portfolio return, $\boldsymbol{\pi}$ and $\mathbf{X}$ are $n \times 1$ vectors of weights and excess returns, respectively, and $\mathbf{1}^{T} \boldsymbol{\pi}=1$, where $\mathbf{1}$ is vector of $n$ ones. $E(R)=\pi^{T} \boldsymbol{\mu}$, where $\boldsymbol{\mu}^{T}=\left(\mu_{1}, \mu_{2}, \ldots, \mu_{n}\right)$ is the vector of expected returns and $\operatorname{Var}(R)=\pi^{T} \Sigma \pi$, where $\Sigma$ is the $n \times n$ covariance matrix of $\mathbf{X}$, the vector of portfolio excess returns. Let us notice that the mean-variance model is the balanced difference between the expectation of returns, which measures an average asset profit, and the variance of returns, which measures the associated risk. Maximization of $M V(R)$ is motivated, therefore, by maximization of average asset profit and minimization of asset risk. Let us also notice that in the case when short selling is possible, the mean-variance OPS problem has an analytic explicit-form solution, because it reduces to a classical quadratic programming problem. 
Another key approach in the OPS theory is the maximization of the Sharpe ratio (see, for instance, Sharpe 1998), which was introduced in Sharpe (1966), and has the form

$$
S(R)=\frac{E(R)-R_{f}}{\sqrt{\operatorname{Var}(R)}}
$$

where $R_{f}$ is the risk free rate. Here we have a ratio between expectation of returns and their standard deviation. Again, the maximization of the Sharpe ratio is motivated by maximization of expected profit and minimization of risk. We observe that there is no analytic solution to this maximization problem even for the situation when short selling is permitted.

In this paper, we offer a novel utility measure, which generalizes both the mean-variance, Sharpe measures and many other celebrated risk measures, and represents a much more flexible measure for incorporating $E(R)$ and $\operatorname{Var}(R)$,

$$
\mathcal{F}(\pi)=\mathcal{F}(\pi ; t, p, v)=t\left(\frac{p(E(R))}{v(\operatorname{Var}(R))}\right) .
$$

Here $t(x), p(x)$ and $v(x)$ are differentiable functions, where $t(x)$ is a monotonic increasing function, i.e., $t^{\prime}(x)>0, p(x)$ is a positive convex or concave function, and $v(x)$ is defined on $[0, \infty)$ and is positive on $(0, \infty)$.

We found an analytical explicit-form solution of the optimization of the general functional $\mathcal{F}(\pi)$ subject to a system of affine equality constraints, when short selling is permitted. The solution takes the following explicit form

$$
\pi^{*}=\pi^{0}+w^{*} \mathbf{z}
$$

where both $\pi^{0} \in R^{n}$ and $\mathbf{z} \in R^{n}$ have explicit closed-forms and they do not depend on the functionals $t, p$, and $v$, which means that these vectors are the same for different OPS models (e.g., the MV and the Sharpe ratio models), while the parameter $w^{*} \in R^{+}$depends on the models that are used.

The paper is organized as follows: In Section 2 we provide an explicit solution to the maximization of (3) subject to (5). In Section 3 we give a numerical illustration of the main results for a special case of the general model. Section 4 provides a conclusion to the paper.

\section{Main Results}

The generalized measure we introduce is based on the maximization of (3) subject to the linear constraints

$$
B \pi=\mathbf{c}, \mathbf{c} \neq \mathbf{0},
$$

where $B=\left(b_{i j}\right)_{i, j=1}^{m, n}$ is $m \times n, m<n$, rectangular matrix of full rank, $\mathbf{c}$ is some $m \times 1$ vector and $\mathbf{0}$ is a vector-column of $m$ zeros. The motivation of such a system of linear constraints arises from models, such as the MV, where the linear constraint $\mathbf{1}^{T} \boldsymbol{\pi}=1$ ascertains that the sum of the weights of the portfolio is equal to one, with positive and negative values corresponding to long and short positions, respectively. More elaborate systems of constraints associated with optimal portfolio selection can be found in Best and Grauer (1990); Landsman (2008b), and in Landsman and Makov (2016). For example, in the case where $\mathbf{c}=\left(1, m_{1}, m_{2}\right)^{T}$ and $B$ is of dimension $3 \times n$ and is equal to

$$
B=\left(\begin{array}{cccccc}
1 & \ldots & \ldots & \ldots & \ldots & 1 \\
\mu_{1} & \ldots & \mu_{j} & 0 & \ldots & 0 \\
0 & \ldots & 0 & \mu_{j+1} & \ldots & \mu_{n}
\end{array}\right),
$$

Equation (5) represents the case when the expected return of a sub-portfolio of size $j$ (the first $j$ returns) is equal to $m_{1}$ and the expected return of a remaining sub-portfolio of size $n-j$ (the remaining $n-j$ returns) is equal to $m_{2}$. 
We show that $\pi^{*}$, the solution of the maximization of (3) subject to (5), is taking an explicit form, which coincides with the solution of the mean-variance problem (16), where the trade-off parameter is $\lambda^{*}=1 /\left(2 w^{*}\right)$, and $w^{*}$ is a solution of an algebraic equation to be presented later.

Before we formulate the main theorem, we begin by introducing certain notations and partitions, which will be helpful in the sequel. Consider the natural partitions of vector $\pi^{T}=\left(\pi_{1}^{T}, \pi_{2}^{T}\right)$ where $\pi_{1}=\left(\pi_{1}, \ldots, \pi_{n-m}\right)^{T}$ and $\pi_{2}=\left(\pi_{n-m+1}, \ldots, \pi_{n}\right)^{T}$, and consider the respective partitions of $\boldsymbol{\mu}^{T}=\left(\boldsymbol{\mu}_{1}^{T}, \boldsymbol{\mu}_{2}^{T}\right), \mathbf{1}^{T}=\left(\mathbf{1}_{1}^{T}, \mathbf{1}_{2}^{T}\right)$ and $\mathbf{0}^{T}=\left(\mathbf{0}_{1}^{T}, \mathbf{0}_{2}^{T}\right)$, where $\mathbf{1}, \mathbf{0}$ are vectors of $n$ ones and zeros, respectively. In a similar way, let us partition the matrix $\Sigma$,

$$
\Sigma=\left(\begin{array}{ll}
\Sigma_{11} & \Sigma_{12} \\
\Sigma_{21} & \Sigma_{22}
\end{array}\right),
$$

and matrix

$$
B=\left(\begin{array}{ll}
B_{21} & B_{22}
\end{array}\right),
$$

where matrices $B_{21}$ and $B_{22}$ are of dimensions $m \times(n-m)$ and $m \times m$, respectively. As matrix $B$ is full rank, suppose, without loss of generality, that matrix $B_{22}$ is non singular.

Furthermore, we define $m \times(n-m)$ and $(n-m) \times m$ matrices

$$
\begin{aligned}
& D_{21}=B_{22}^{-1} B_{21}, \\
& D_{12}=D_{21}^{T},
\end{aligned}
$$

and $(n-m) \times(n-m)$ matrix

$$
Q=\Sigma_{11}-\Sigma_{12} D_{21}-D_{12} \Sigma_{21}+D_{12} \Sigma_{22} D_{21}=\left(q_{i j}\right)_{i, j=1}^{n-m} .
$$

Notice that as $\Sigma$ is positive definite, $Q$ is also positive definite (see Landsman 2008a).

Denote by

$$
\begin{gathered}
\boldsymbol{\Delta}=\boldsymbol{\mu}_{1}-D_{12} \boldsymbol{\mu}_{2}, \\
f_{0}=\mathbf{c}^{T}\left(B \Sigma^{-1} B^{T}\right)^{-1} \mathbf{c}>0, \\
b^{2}=\Delta^{T} Q^{-1} \Delta>0
\end{gathered}
$$

and define functions $u_{1}$ and $u_{2}$

$$
u_{1}(x)=\frac{v^{\prime}(x)}{v(x)}
$$

and

$$
u_{2}(x)=\frac{p^{\prime}(x)}{p(x)}
$$

where $v(x)$ and $p(x)$ are functions in (3).

We note that function $v(x)$ must satisfies the following condition:

(C) The function $h(x)=v\left(a x^{2}+2 q x+r\right)$ is strictly convex on $\mathbb{R}$ for any numbers $a>0, q$, and $r$ such that $q^{2}-$ ar $<0$.

This condition is essentially weaker than a condition of convexity of $v$. For example, $v(x)=\sqrt{x}$ is concave but condition (C) still holds.

Theorem 1. Suppose that $u_{1}(x)>0$ and $u_{2}(x)>0$. If the following equation with respect to $w$,

$$
u_{1}\left(f_{0}+b^{2} w^{2}\right) w=\frac{1}{2} u_{2}\left(\boldsymbol{\mu}_{2}^{T} B_{22}^{-1} \mathbf{c}+\Delta^{T} Q^{-1}\left(D_{12} \Sigma_{22}-\Sigma_{12}\right) B_{22}^{-1} \mathbf{c}+w b^{2}\right),
$$


has a positive solution $w^{*}$, this solution is unique and the problem of maximization

$$
\mathcal{F}(\boldsymbol{\pi})=t\left(\frac{p\left(\pi^{T} \boldsymbol{\mu}\right)}{v\left(\boldsymbol{\pi}^{T} \sum \pi\right)}\right) \rightarrow \max
$$

subject to (5) coincides with the solution of the mean-variance optimal problem, where the trade-off parameter $\lambda$ takes the value

$$
\lambda^{*}=\frac{1}{2 w^{*}}
$$

In other words the solution to (11) is given by

$$
\pi^{*}=\pi^{0}+w^{*} \mathbf{z}
$$

Here

$$
\pi^{0}=\Sigma^{-1} B^{T}\left(B \Sigma^{-1} B^{T}\right)^{-1} \mathbf{c}
$$

and

$$
\mathbf{z}=\Sigma^{-1} \boldsymbol{\mu}-\Sigma^{-1} B^{T}\left(B \Sigma^{-1} B^{T}\right)^{-1} B \Sigma^{-1} \boldsymbol{\mu} .
$$

The proof of the Theorem is given in the Appendix A.

This Theorem essentially generalizes Theorem 1 of Landsman and Makov (2016), where a special case was considered when $t(x)=\log x, p(x)=\exp (x), v(x)=\exp (\lambda s(x))$. Notice that in Landsman and Makov (2016) vector $\mathbf{z}$ was presented in a less convenient form than that of (A19).

Theorem 1 may have the following economic interpretation: it expresses the competition between the minimum variance portfolio $\left(\pi^{0}\right)$ and vector $\mathbf{z}$; these vectors appear in the classical mean variance portfolio and do not depend on the choice of the utility function and tuning parameter $w^{*}$, which reflects by investor's preference. From expression of vector z, (A19), immediately follows that

$$
B \mathbf{z}=\mathbf{0}
$$

Then, for the special case, when $m=1$, matrix $B=\mathbf{1}^{T}$ and $c=1$ we have $\mathbf{1}^{T} \mathbf{z}=\mathbf{0}$ and the vector $\mathbf{z}$ can be interpreted as a self-financing portfolio in the sense that long-positions are financed by corresponding short positions (see, for instance, Panjer and Boyle (1998), sct. 8.2.1). This case is considered in more details below, in Remark 1.

We now state a property related to the efficient frontier of the class of utility measures generated by (3). First of all we notice that for our utility function (3)

$$
\frac{\partial \mathcal{F}}{\partial E}=t^{\prime}(\cdot) \frac{p^{\prime}(x)}{v(y)}>0, \frac{\partial \mathcal{F}}{\partial V a r}=-t^{\prime}(\cdot) \frac{p(E)}{v(\text { Var })^{2}} v^{\prime}(\text { Var })<0 .
$$

These inequalities are very natural and important. They say that utility function $\mathcal{F}$ is increased with respect to $E(R)$ and decreased with respect to $\operatorname{Var}(R)$.

Corollary 1. The efficient frontier of the optimization problem (3) subject to (5) coincides with or belongs to the efficient frontier of the classical mean-variance utility function and has a parabolic form (see below (A22) and (A23)).

The proof of the Corollary is given in the Appendix A.

Remark 1. In the special case when $m=1$, matrix $B=\mathbf{1}^{T}$ and $c=1$, we can conclude that

$$
\boldsymbol{\pi}^{0}=\frac{\Sigma^{-1} \mathbf{1}}{\mathbf{1}^{T} \Sigma^{-1} \mathbf{1}}
$$




$$
\mathbf{z}=\Sigma^{-1} \boldsymbol{\mu}-\frac{\boldsymbol{\mu}^{T} \Sigma^{-1} \mathbf{1}}{\mathbf{1}^{T} \Sigma^{-1} \mathbf{1}} \Sigma^{-1} \mathbf{1}
$$

which conform with Panjer and Boyle (1998, (8.2.7)-(8.2.9)). See also (Steinbach 2001, Theorem 1.4). From (A22) follows that elements of the efficient frontier are

$$
\mu_{0}=\frac{\mathbf{1}^{T} \Sigma^{-1} \boldsymbol{\mu}}{\mathbf{1}^{T} \Sigma^{-1} \mathbf{1}}, \mu_{\mathbf{z}}=\boldsymbol{\mu}^{T} \Sigma^{-1} \boldsymbol{\mu}-\frac{\left(\mathbf{1}^{T} \Sigma^{-1} \boldsymbol{\mu}\right)^{2}}{\mathbf{1}^{T} \Sigma^{-1} \mathbf{1}}, \sigma_{0}^{2}=\frac{1}{\mathbf{1}^{T} \Sigma^{-1} \mathbf{1}}, \sigma_{\mathbf{z}}^{2}=\mu_{\mathbf{z}}
$$

The orthogonality property (A20) for this case was proved by Best and Best and Grauer (1990).

\subsection{Optimal Portfolios for Special Cases}

The investigated generalized measure, includes, as special cases, key measures employed in risk management.

We now show some special cases of the generalized measure $\mathcal{F}(\pi)$ :

Special case 1: Sharpe ratio (SR). For $t(x)=x, p(x)=x-R_{f}$ and $v(x)=\sqrt{x}$, (3) becomes the Sharpe ratio measure (2)

$$
S(R)=\mathcal{F}\left(\pi ; x, x-R_{f}, \sqrt{x}\right)=\frac{\pi^{T} \mu-R_{f}}{\sqrt{\pi^{T} \sum \pi}}
$$

Special case 2: Generalized Sharpe ratio (GSR). We introduce a generalized Sharpe ratio

$$
S_{\beta}(R)=\mathcal{F}\left(\pi ; x, x-R_{f}, x^{\beta}\right)=\frac{E(R)-R_{f}}{(\operatorname{Var}(R))^{\beta}},
$$

which is a special case of (3), for which $t(x)=x, p(x)=x-R_{f}$ and $v(x)=x^{\beta}$, and found an analytic solution of the corresponding optimization problem for $\beta \geq 1 / 2$. The economic justification for introducing this utility function can be explained by investor's wish to allow faster decrease in the utility when the variance increases. In this section, we show that as the investors are more risk-averse they should consider larger value of $\beta$, the power function parameter, so $\beta$ is subjective for each investor. The $\beta$ parameter can be interpreted as a new risk aversion parameter, which was absent in the classical Sharpe ratio. This risk measure is much more flexible than the classical Sharpe ratio for which $\beta=1 / 2$. In Section 2.1.4 we explain this in more details.

Special case 3: Mean Variance (MV). In this case, $t(x)=\ln (x), p(x)=e^{x}$ and $v(x)=e^{\lambda x}, \lambda>0$, and the goal function (3) takes the form

$$
\mathcal{F}\left(\pi ; \ln (x), e^{x}, e^{\lambda x}\right)=\pi^{T} \mu-\lambda \pi^{T} \Sigma \pi
$$

Special case 4: Mean Standard Deviation (MSD). In the case $t(x)=\ln (x), p(x)=e^{x}$ and $v(x)=e^{\lambda \sqrt{x}}, \lambda>0,(3)$ has the form

$$
\mathcal{F}\left(\pi ; \ln (x), e^{x}, e^{\lambda_{q} \sqrt{x}}\right)=\pi^{T} \mu-\beta \sqrt{\pi^{T} \Sigma \pi} .
$$

This functional arises in risk management and actuarial theory. For instance, the value at risk and the expected shortfall takes the form (17) for returns having elliptical distribution (see, for example, Landsman and Valdez (2003)). 


\subsubsection{Optimal Portfolio Selection With MV Measure}

This case was implicitly considered in the proof of Theorem 1. Recall that for MV $t(x)=\ln (x)$, $p(x)=e^{x}$ and $v(x)=e^{\lambda x}, \lambda>0$, and the functions $u_{1}$ and $u_{2}$ take the forms $u_{1}(x)=\lambda, u_{2}(x)=1$. Therefore, Equation (10) simply reduces to

$$
w^{*}=\frac{1}{2 \lambda} .
$$

It is clear that $w^{*}$ is positive and unique, and the point of maximum $\pi^{*}$ is (12) with $w^{*}=1 /(2 \lambda)$. Clearly, this optimal solution is only meaningful if $\lambda$ is specified. Notice that the MV measure can be also computed explicitly from the main Theorem of Landsman and Makov (2016), but, the solution in Landsman and Makov (2016) is much more complex in its form than Theorem 1. This proposed solution well conforms with Panjer and Boyle (1998, (8.2.7)-(8.2.9)).

\subsubsection{Optimal Portfolio Selection With MSD}

As was discussed in the introduction, for the MSD utility function, $t(x)=\ln (x), p(x)=e^{x}$ and $v(x)=e^{\beta \sqrt{x}}, \beta>0$ and the main functional reduces to the form

$$
\mathcal{F}(\pi)=\pi^{T} \mu-\beta \sqrt{\pi^{T} \Sigma \pi},
$$

which has a form of a combination of linear functional and a square root of a quadratic functional. The optimal problem with this functional was considered in details in Landsman (2008a, 2008b). Here we only show how the solution of this problem can be obtained from Theorem 1 .

We note that the functions $u_{1}$ and $u_{2}$ have the form

$$
u_{1}(x)=\beta \frac{1}{2 \sqrt{x}}, u_{2}(x)=1,
$$

and Equation (10), which reduces to

$$
\beta \frac{1}{2{\sqrt{f_{0}+b^{2} w^{2}}}^{2}} w=\frac{1}{2}
$$

has the positive solution,

$$
w^{*}=\sqrt{\frac{f_{0}}{\beta^{2}-b^{2}}}=\sqrt{\frac{\mathbf{c}^{T}\left(B \Sigma^{-1} B^{T}\right) \mathbf{c}}{\beta^{2}-\Delta^{T} Q^{-1} \Delta}},
$$

iff $\beta^{2}>b^{2}=\Delta^{T} Q^{-1} \Delta$.

Now, substituting $w^{*}$ into (12), we finally get the maximization solution $\mathbf{x}^{*}$, which conforms with Theorem 1 in Landsman (2008b). Applying Theorem 1 we can say that the optimal portfolio coincides with mean-variance portfolio when the trade off parameter is

$$
\lambda^{*}=\frac{1}{2} \sqrt{\frac{\beta^{2}-\Delta^{T} Q^{-1} \Delta}{\mathbf{c}^{T}\left(B \Sigma^{-1} B^{T}\right) \mathbf{c}}} .
$$

Note that although the optimal solution can be derived, in principle, from the mean-variance utility function, the choice of $\lambda$ remains a guess, unless calculated $\left(\lambda^{*}\right)$ using the main theorem of this paper. 


\subsubsection{Optimal Portfolio Selection With Sharpe Ratio}

Recall that the Sharpe ratio functional has the form

$$
\mathcal{F}(\pi)=\frac{\pi^{T} \mu-R_{f}}{\sqrt{\pi^{T} \Sigma \pi}}
$$

It is clear that for the solution of the maximization problem it is enough to consider $\pi^{T} \mu>R_{f}$. Then $t(x)=x, v(x)=x^{1 / 2}, x>0$ and $p(x)=x-R_{f}, x>R_{f}$. Consequently functions $u_{1}$ and $u_{2}$ simply have the form

$$
u_{1}(x)=\frac{1}{2 x}, x>0, u_{2}(x)=\frac{1}{x-R_{f}}, x>R_{f}
$$

Thus Equation (10) reduces to algebraic equation of the first degree and the unique solution $w^{*}$ is

$$
w^{*}=\frac{\mathbf{c}^{T}\left(B \Sigma^{-1} B^{T}\right)^{-1} \mathbf{c}}{\mu_{2}^{T} B_{22}^{-1} \mathbf{c}+\Delta^{T}\left(Q^{-1}\left(D_{12} \Sigma_{22}-\Sigma_{12}\right) B_{22}^{-1} \mathbf{c}\right)-R_{f}} .
$$

Now since $\mathbf{c}^{T}\left(B \Sigma^{-1} B^{T}\right)^{-1} \mathbf{c}>0$, it is sufficient to determine that iff

$$
\boldsymbol{\mu}_{2}^{T} B_{22}^{-1} \mathbf{c}+\Delta^{T}\left(Q^{-1}\left(D_{12} \Sigma_{22}-\Sigma_{12}\right) B_{22}^{-1} \mathbf{c}\right)>R_{f}
$$

the solution of (18) is positive and then we obtain the explicit maximum solution $\pi^{*}$ which has the form given in (12).

Hitherto, an optimal portfolio selection for the Sharpe ratio utility function has been unavailable. We establish that the optimal solution is a special case of the mean-variance utility function, where, using (18), $\lambda^{*}$ can now be calculated to be $\lambda^{*}=\frac{1}{2 w^{*}}$.

\subsubsection{Optimal Portfolio With Generalized Sharpe Ratio}

The functional of the GSR was introduced previously, as follows:

$$
\mathcal{F}(\pi)=\frac{\pi^{T} \mu-R_{f}}{\left(\pi^{T} \Sigma \pi\right)^{\beta}}, \beta \geq \frac{1}{2}
$$

In this functional, $v(x)=x^{\beta}$, where $\beta \geq \frac{1}{2}$ is a power function. Recall $t(x)=x$, and $p(x)=x-R_{f}, x>R_{f}$. Now we consider the case where $\beta>\frac{1}{2}$, (the case $\beta=1 / 2$ was considered in the previous subsection). Then functions $u_{1}$ and $u_{2}$ take the form

$$
u_{1}(x)=\frac{\beta}{x}, x>0, u_{2}(x)=\frac{1}{x-R_{f}}, x>R_{f},
$$

and Equation (10) reduces to a quadratic equation with respect to $w$

$$
b^{2}\left(\beta-\frac{1}{2}\right) w^{2}+\beta \delta w-\frac{1}{2} f_{0}=0,
$$

where $\delta=\mu_{2}^{T} B_{22}^{-1} \mathbf{c}+\Delta^{T} Q^{-1}\left(D_{12} \Sigma_{22}-\Sigma_{12}\right) B_{22}^{-1} \mathbf{c}-R_{f}$. Notice that (20) has the unique positive solution.

$$
w^{*}=\frac{-\beta \delta+\sqrt{\beta^{2} \delta^{2}+2 b^{2} f_{0}\left(\beta-\frac{1}{2}\right)}}{2 b^{2}\left(\beta-\frac{1}{2}\right)},
$$

and when $\delta>0$ the maximum solution $\mathbf{x}^{*}$ takes the form of (12). 
We now return to the economic interpretation of this functional, comparing it with Sharpe ratio. In fact, returning to the arguments given after Theorem 1 we obtain that

$$
\frac{\partial S_{\beta}}{\partial \operatorname{Var}}=-\beta \frac{E R-R_{f}}{\operatorname{Var}(R)^{\beta+1}}<-\frac{1}{2} \frac{E R-R_{f}}{\operatorname{Var}(R)^{3 / 2}}=\frac{\partial S_{\beta=1 / 2}}{\partial \operatorname{Var}}, \beta>1 / 2 .
$$

For $\beta>1 / 2$, the speed of decrease of the generalized Sharpe ratio $S_{\beta}$ in Var is higher than that of decrease of the Sharpe ratio $S_{\beta=1 / 2}$.

As for the efficient frontier, we conclude from (21) that the efficient frontier corresponding to the generalized and classical Sharpe ratios belongs to the efficient frontier corresponding to the mean-variance model (See proof of Corollary 1), with the following set showing the relation between the MV risk aversion parameter, $\lambda$, and the generalized Sharpe ratio risk aversion parameter, $\beta$,

$$
\Gamma=\left\{\lambda \mid \frac{b^{2}\left(\beta-\frac{1}{2}\right)}{-\beta \delta+\sqrt{\beta^{2} \delta^{2}+2 b^{2} f_{0}\left(\beta-\frac{1}{2}\right)}}, \beta \geq 1 / 2\right\}=\left[\delta /\left(2 f_{0}\right), \infty\right) \subset R_{+} .
$$

Applying Theorem 1 we can say that the optimal portfolio coincides with mean-variance portfolio when the trade-off parameter is

$$
\lambda^{*}=\frac{b^{2}\left(\beta-\frac{1}{2}\right)}{-\beta \delta+\sqrt{\beta^{2} \delta^{2}+2 b^{2} f_{0}\left(\beta-\frac{1}{2}\right)}} .
$$

The following Figure shows the relation between the $\lambda^{*}$ and $\beta$. As we can see from Figure 1 , $\lambda^{*}$ increased almost proportionally when $\beta$ increases.

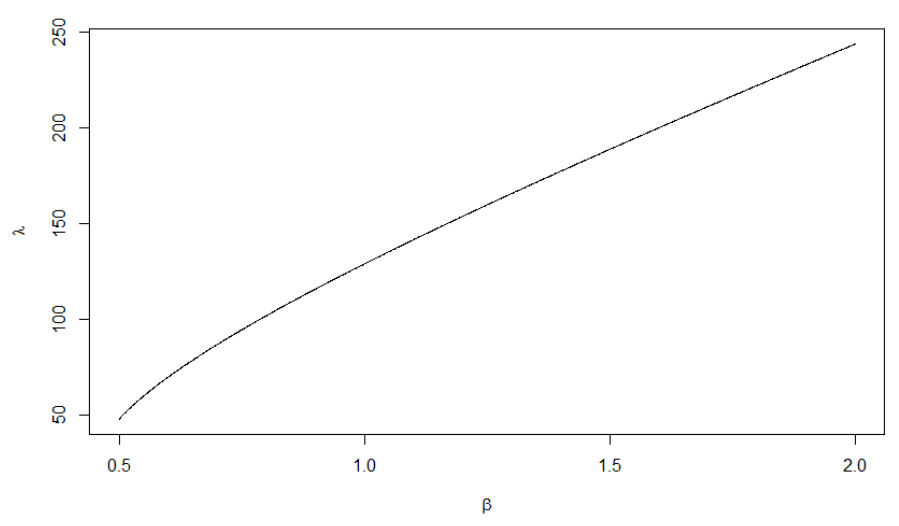

Figure 1. The relations between $\lambda^{*}$ and $\beta$.

Note that acknowledging the fact that the optimal solution corresponds to a solution derived from the mean-variance utility function is not enough since the value of the trade of $\lambda$ remains unknown. The result provided here allows us to calculate its value.

\section{Numerical Illustrations}

We illustrate the results of the OPS problem for the mean standard deviation utility function and for special cases of the generalized Sharpe ratio

$$
S_{\beta}=\frac{\pi^{T} \boldsymbol{\mu}-R_{f}}{\left(\pi^{T} \Sigma \pi\right)^{\beta}}
$$

with $\beta=0.5,1,2$, and free rate $R_{f}=0.00016$ for a portfolio of 10 stocks from NASDAQ: Facebook, Intel, Frontier, Micron, Apple, Qualcomm, Sirius, Applied Materials, Cisco and Yahoo, for a three 
month period of time, from 4 January 15 to 7 January 15-daily returns (For the data, see http: //www.nasdaq.com/). Here $\mu$ is the vector of expected returns and $\Sigma$ is the covariance matrix or returns presented in Tables 1 and 2, respectively. Furthermore, we assume the traditional linear constraint $\mathbf{1}^{T} \pi=1$.

Then, using formula (24) we can calculate trade off parameters $\lambda^{*}$ and then obtain the vectors of solutions, for each model, are given in Table 3.

Table 1. Expected returns.

\begin{tabular}{lccccc}
\hline Stock & Facebook & Intel & Frontier & Micron & Apple \\
\hline Mean & 0.000868097 & -0.000608624 & -0.006684089 & -0.006902419 & $-6.1631 \times 10^{-5}$ \\
Stock & Qualcomm & Sirius & App. Mat. & Cisco & Yahoo \\
Mean & 0.001046047 & 0.000763278 & 0.002049615 & $-2.57636 \times 10^{-5}$ & 0.001925747 \\
\hline
\end{tabular}

Table 2. Covariance matrix of the returns.

\begin{tabular}{cccccc}
\hline & Facebook & Intel & Frontier & Micron & Apple \\
\hline Facebook & 0.000175 & 0.000038 & 0.000054 & 0.000063 & -0.000014 \\
Intel & 0.000038 & 0.000174 & 0.000075 & 0.000213 & -0.000014 \\
Frontier & 0.000054 & 0.000075 & 0.000685 & 0.000031 & -0.000001 \\
Micron & 0.000063 & 0.000213 & 0.000031 & 0.001031 & 0.000048 \\
Apple & -0.000014 & -0.000014 & -0.000001 & 0.000048 & 0.000124 \\
\hline & Qualcomm & Sirius & App. Mat. & Cisco & Yahoo \\
\hline Facebook & 0.000029 & -0.000015 & -0.000019 & 0.000006 & 0.000010 \\
Intel & 0.000030 & 0.000086 & 0.000024 & 0.000028 & 0.000047 \\
Frontier & 0.000084 & -0.000014 & 0.000071 & 0.000050 & 0.000095 \\
Micron & 0.000027 & 0.000023 & 0.000050 & -0.000002 & 0.000047 \\
Apple & -0.000002 & 0.000012 & 0.000015 & -0.000010 & 0.000046 \\
\hline & Qualcomm & Sirius & App. Mat. & Cisco & Yahoo \\
\hline Qualcomm & 0.000108 & 0.000054 & 0.000038 & 0.000054 & 0.000075 \\
Sirius XM & 0.000054 & 0.000097 & 0.000049 & 0.000044 & 0.000060 \\
App. Mat. & 0.000038 & 0.000049 & 0.000235 & 0.000046 & 0.000086 \\
Cisco & 0.000054 & 0.000044 & 0.000046 & 0.000084 & 0.000037 \\
Yahoo & 0.000075 & 0.000060 & 0.000086 & 0.000037 & 0.000316 \\
\hline
\end{tabular}

Table 3. The solutions $\pi^{*}$ for different models: Mean standard deviation (MSD), Sharpe ratio (SR) and generalized SR.

\begin{tabular}{ccccccc}
\hline Utility Function & $\lambda^{*}$ & $\pi_{1}^{*}$ & $\pi_{2}^{*}$ & $\pi_{3}^{*}$ & $\pi_{4}^{*}$ & $\pi_{5}^{*}$ \\
\hline $\boldsymbol{\pi}^{T} \boldsymbol{\mu}-\sqrt{\pi^{T} \Sigma \pi}$ & 61.78 & -0.282 & 1.938 & -0.496 & -0.432 & 0.809 \\
\hline$\frac{\boldsymbol{\pi}^{T} \boldsymbol{\mu}-R_{f}}{\left(\boldsymbol{\pi}^{T} \Sigma \pi\right)^{0.5}}$ & 47.6 & -0.402 & 2.418 & -0.627 & -0.542 & 0.937 \\
$\frac{\boldsymbol{\pi}^{T} \boldsymbol{\mu}-R_{f}}{\left(\pi^{T} \Sigma \pi\right)}$ & 128.8 & -0.071 & 1.094 & -0.264 & -0.238 & 0.584 \\
$\frac{\pi^{T} \boldsymbol{\mu}-R_{f}}{\left(\boldsymbol{\pi}^{T} \Sigma \pi\right)^{2}}$ & 243.7 & 0.019 & 0.727 & -0.163 & -0.154 & 0.486 \\
\hline Utility Function & $\pi_{6}^{*}$ & $\pi_{7}^{*}$ & $\pi_{8}^{*}$ & $\pi_{9}^{*}$ & $\pi_{10}^{*}$ & \\
\hline$\pi^{T} \boldsymbol{\mu}-\sqrt{\pi^{T} \Sigma \pi}$ & 1.382 & -2.613 & 0.419 & 0.314 & -0.0391 & \\
\hline$\frac{\pi^{T} \boldsymbol{\mu}-R_{f}}{\left(\boldsymbol{\pi}^{T} \Sigma \pi\right)^{0.5}}$ & 1.731 & -3.313 & 0.516 & 0.319 & -0.0377 & \\
$\frac{\pi^{T} \boldsymbol{\mu}-R_{f}}{\left(\pi^{T} \Sigma \pi\right)}$ & 0.766 & -1.382 & 0.247 & 0.305 & -0.041 & \\
$\frac{\pi^{T} \boldsymbol{\mu}-R_{f}}{\left(\boldsymbol{\pi}^{T} \Sigma \pi\right)^{2}}$ & 0.499 & -0.847 & 0.173 & 0.301 & -0.042 & \\
\hline
\end{tabular}


In the following Figure we present the spectrum of optimal portfolios, given in Table 3.

From Figure 2 it is evident that the amplitude of the weights decreases with increasing values of $\beta$. This well conforms with the graph of $\lambda^{*}$, presented in Figure 1. As a result it can be observed that the portfolio become more robust when $\beta$ increases. This agrees with the expression of portfolio variance, given in the second formula of (A21), associated with the risk of the portfolio. According to Figure 2, the stocks: Intel, Apple, Qualcomm, App. Mat. and Cisco should be invested in a long position, while the other stocks should be invested in a short position. Furthermore, all of the models are in agreement that the stocks with the largest impact on the portfolio are Intel and Sirius. Table 3, presents the solutions $\pi^{*}$ for the different models: MSD, SR and GSR, and together with the graphical illustration (Figure 2) show that all of the mean-variance-based risk measures share the same patterns in terms of higher/lower gains, so the main difference between them is the amplitude.

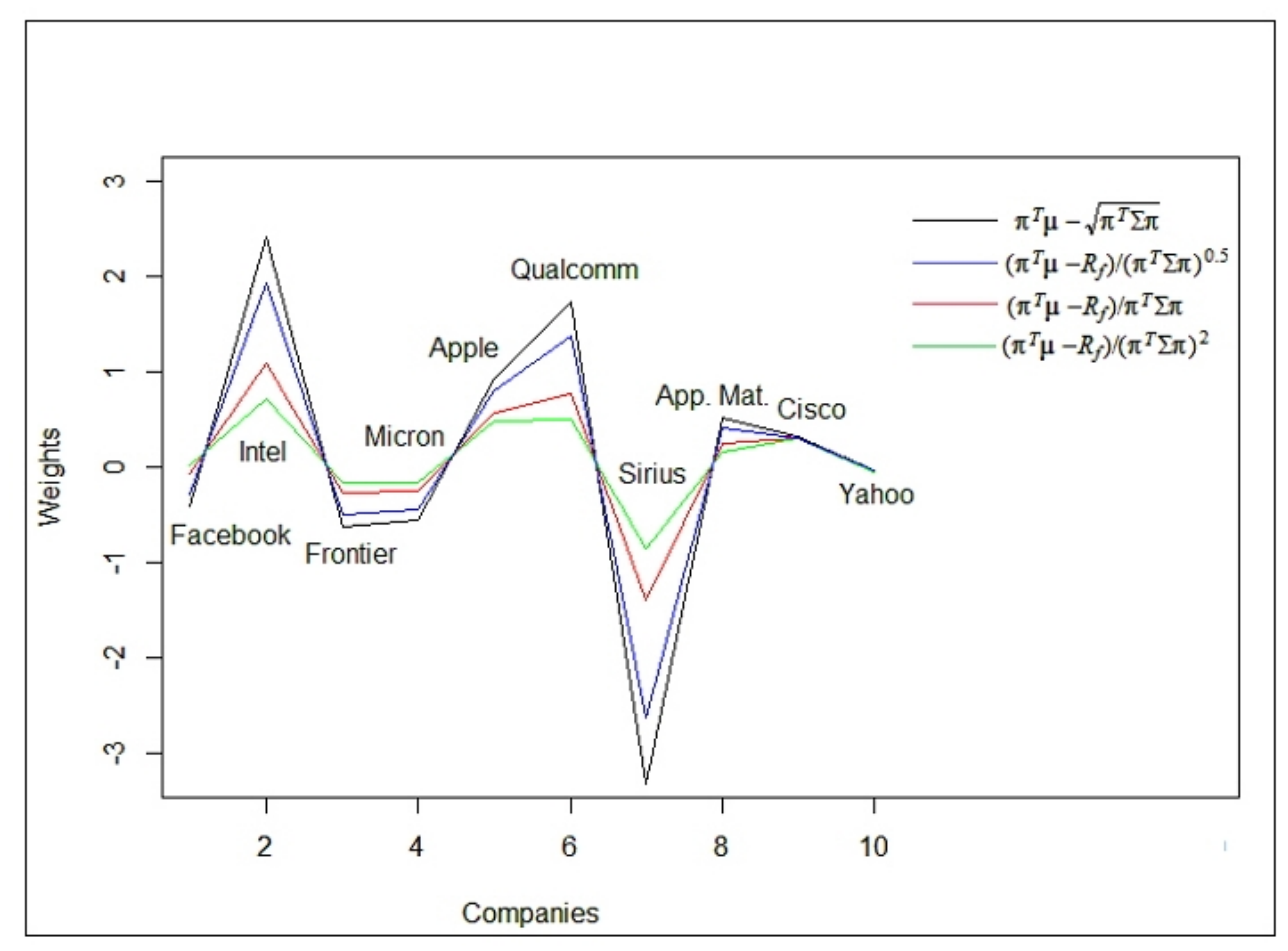

Figure 2. Optimal portfolios for Mean standard deviation (MSD) and generalized Sharpe ratio, $\beta=0.5,1,2$, utility functions.

This numerical illustration shows that investors with a more risk-averse character should consider larger values of $\beta$ than the Sharpe ratio measure. Therefore, the flexibility of the generalized Sharpe ratio is governed by the different values of $\beta$, the subjective parameter corresponding to the level of risk aversion. The generalized Sharpe ratio shows how to implement, naturally, a risk aversion parameter into the framework of the standard Sharpe ratio (2).

\section{Conclusions}

In this paper, we suggested a novel generalized optimal portfolio selection utility measure which incorporates many popular portfolio selection utility measures as special cases. We further provided an explicit solution to this new utility measure, thus reestablishing existing optimal solutions to portfolio selection and offered closed-form solutions to optimal portfolio selections based on new utility measures which hitherto have not been investigated. We note that while the optimal solution for each of these utility functions can be obtained using the mean-variance utility function, the corresponding $\lambda$ is simply unavailable without the methodology presented here. Furthermore, we demonstrated our 
results using a portfolio consisting ten stocks from NASDAQ in a period of three months and analyzed the results for the mean standard deviation utility function and for the generalized Sharpe ratio.

Acknowledgments: We would like to thank the anonymous referees for their useful comments. This research was supported by the Israel Science Foundation (grant No. 1686/17). The authors also wish to thank the Israel Zimmerman Foundation for the Study of Banking and Finance for financial support.

Author Contributions: The authors contribute equally to this article.

Conflicts of Interest: The authors declare no conflict of interest.

\section{Appendix A. Proofs}

Proof of Theorem 1. We first note that since $p(x)$ is a convex or concave function, $p\left(\boldsymbol{\mu}^{T} \boldsymbol{\pi}\right)$ is also a convex or concave function. We further define vector

$$
\mathbf{d}_{2}=B_{22}^{-1} \mathbf{c},
$$

then, by (5) and (6) it follows that

$$
\pi=\left(\boldsymbol{\pi}_{1}^{T}, \mathbf{d}_{2}^{T}-\boldsymbol{\pi}_{1}^{T} D_{12}\right)^{T}
$$

and straightforwardly

$$
\pi^{T} \Sigma \pi=\pi_{1}^{T} Q \pi_{1}+2 \mathbf{d}_{2}^{T}\left(\Sigma_{21}-\Sigma_{22} D_{21}\right) \pi_{1}+\mathbf{d}_{2}^{T} \Sigma_{22} \mathbf{d}_{2} .
$$

Then the objective function

$$
\mathcal{F}(\boldsymbol{\pi})=g\left(\boldsymbol{\pi}_{1}\right)=t\left(\frac{p\left(\boldsymbol{\mu}_{2}^{T} \mathbf{d}_{2}+\Delta^{T} \boldsymbol{\pi}_{1}\right)}{v\left(\boldsymbol{\pi}_{1}^{T} Q \boldsymbol{\pi}_{1}+2 \mathbf{d}_{2}^{T}\left(\Sigma_{21}-\Sigma_{22} D_{21}\right) \boldsymbol{\pi}_{1}+\mathbf{d}_{2}^{T} \Sigma_{22} \mathbf{d}_{2}\right)}\right),
$$

is a function of $n-m$ variables $\pi_{1}=\left(\pi_{1}, \ldots, \pi_{n-m}\right)^{T}$ and the problem reduces to the problem of finding the unconditional maximum

$$
\max _{\pi_{1} \in R^{n-m}} g\left(\pi_{1}\right) .
$$

Denoting a $1 \times(n-m)$ vector of derivatives with the following notation

$$
\frac{d}{d \pi_{1}}=\left(\frac{d}{d \pi_{1}}, \frac{d}{d \pi_{2}}, \ldots, \frac{d}{d \pi_{n-m}}\right)^{T}
$$

we clearly seek, taking into account (8) and (9), that

$$
\begin{aligned}
\frac{d}{d \boldsymbol{\pi}_{1}} g\left(\boldsymbol{\pi}_{1}\right) & =\frac{d}{d \boldsymbol{\pi}_{1}}\left(t\left(\frac{p}{v}\right)\right)=t^{\prime} \cdot p \cdot v^{-1} \cdot\left[\Delta u_{2}\left(\boldsymbol{\mu}_{2}^{T} \mathbf{d}_{2}+\Delta^{T} \boldsymbol{\pi}_{1}\right)\right. \\
& -2\left(Q \boldsymbol{\pi}_{1}+\left(\Sigma_{12}-D_{12} \Sigma_{22}\right) \mathbf{d}_{2}\right) u_{1}\left(\boldsymbol{\pi}_{1}^{T} Q \boldsymbol{\pi}_{1}\right. \\
& \left.\left.+2 \mathbf{d}_{2}^{T}\left(\Sigma_{21}-\Sigma_{22} D_{21}\right) \boldsymbol{\pi}_{1}+\mathbf{d}_{2}^{T} \Sigma_{22} \mathbf{d}_{2}\right)\right] \\
& =\mathbf{0}_{1} .
\end{aligned}
$$

Using (8), it is clear that this system of equations can be rewritten in the form

$$
\frac{1}{2} \Delta u_{2}\left(\mu_{2}^{T} \mathbf{d}_{2}+\Delta^{T} \pi_{1}\right)=u_{1}\left(\pi_{1}^{T} Q \pi_{1}+2 \mathbf{d}_{2}^{T}\left(\Sigma_{21}-\Sigma_{22} D_{21}\right) \pi_{1}+\mathbf{d}_{2}^{T} \Sigma_{22} \mathbf{d}_{2}\right) \cdot\left(Q \pi_{1}+\left(\Sigma_{12}-D_{12} \Sigma_{22}\right) \mathbf{d}_{2}\right),
$$


and we denote the solution of (A4) as $\pi_{1}^{*}$. In Landsman (2008b) it was shown that as a solution of the quadratic programming problem

$$
\pi^{0}=\arg \min _{B \pi=c} \pi^{T} \Sigma \pi=\Sigma^{-1} B^{T}\left(B \Sigma^{-1} B^{T}\right)^{-1} \mathbf{c} .
$$

Then from (A2) we can represent $\pi^{0}$ as a partition

$$
\pi^{0}=\left(\pi_{1}^{0 T}, \mathbf{d}_{2}^{T}-\pi_{1}^{0 T} D_{12}\right)^{T}
$$

As vector $\pi^{0}$ is a point of minimum of quadratic functional $\pi^{T} \Sigma \pi$, from (A3) it follows immediately that $\pi_{1}^{0}$ is the solution of the following equation

$$
Q \pi_{1}^{0}+\left(\Sigma_{12}-D_{12} \Sigma_{22}\right) \mathbf{d}_{2}=\mathbf{0}_{1}
$$

We will now seek $\pi_{1}^{*}$ in the form $\pi_{1}^{*}=\pi_{1}^{0}+\mathbf{y}^{*}$, where $\mathbf{y}^{*}=\left(y_{1}^{*}, \ldots, y_{n-m}^{*}\right)^{T}$ is $(n-m)-$ dimensional vector. Then, taking into account (A5), we straightforwardly obtain from (A4),

$$
\begin{aligned}
& u_{1}\left(\boldsymbol{\pi}_{1}^{* T} Q \boldsymbol{\pi}_{1}^{*}+2 \mathbf{d}_{2}^{T}\left(\Sigma_{21}-\Sigma_{22} D_{21}\right) \boldsymbol{\pi}_{1}^{*}+\mathbf{d}_{2}^{T} \Sigma_{22} \mathbf{d}_{2}\right) \mathbf{y}^{*} \\
& =\frac{1}{2} Q^{-1} \Delta u_{2}\left(\boldsymbol{\mu}_{2}^{T} \mathbf{d}_{2}+\Delta^{T}\left(\pi_{1}^{0}+\mathbf{y}^{*}\right)\right) .
\end{aligned}
$$

We represent a $(n-m) \times(n-m)$ matrix $Q^{-1}$ as follows:

$$
Q^{-1}=\left(\begin{array}{l}
Q_{1}^{-1} \\
Q_{2}^{-1}
\end{array}\right),
$$

where $Q_{1}^{-1}$ is the first row of $Q^{-1}$ and $Q_{2}^{-1}$ consists of the remaining $(n-m-1)$ rows of $Q^{-1}$. As matrix $Q$ is positive definite, $Q^{-1}$ is also positive definite, so there exists a raw $r$ of matrix $Q^{-1}$ such that $Q_{r}^{-1} \Delta \neq 0, r \in\{1, \ldots, n-m\}$. Suppose, without loss of generalization, that $Q_{1}^{-1} \Delta \neq 0$. Then from (A6) follows the system of $n-m$ equations

$$
\left\{\begin{array}{c}
u_{1}\left(\boldsymbol{\pi}_{1}^{* T} Q \boldsymbol{\pi}_{1}^{*}+2 \mathbf{d}_{2}^{T}\left(\Sigma_{21}-\Sigma_{22} D_{21}\right) \pi_{1}^{*}+\mathbf{d}_{2}^{T} \Sigma_{22} \mathbf{d}_{2}\right) y_{1}^{*} \\
=\frac{1}{2} Q_{1}^{-1} \Delta u_{2}\left(\boldsymbol{\mu}_{2}^{T} \mathbf{d}_{2}+\Delta^{T}\left(\mathbf{x}_{1}^{0}+\mathbf{y}^{*}\right)\right) \\
u_{1}\left(\boldsymbol{\pi}_{1}^{* T} Q \pi_{1}^{*}+2 \mathbf{d}_{2}^{T}\left(\Sigma_{21}-\Sigma_{22} D_{21}\right) \pi_{1}^{*}+\mathbf{d}_{2}^{T} \Sigma_{22} \mathbf{d}_{2}\right) \mathbf{y}_{-1}^{*} \\
=\frac{1}{2} Q_{2}^{-1} \Delta u_{2}\left(\boldsymbol{\mu}_{2}^{T} \mathbf{d}_{2}+\Delta^{T}\left(\boldsymbol{\pi}_{1}^{0}+\mathbf{y}^{*}\right)\right)
\end{array}\right.
$$

where $y_{1}^{*}$ is the first element of vector $\mathbf{y}^{*}$, and $\mathbf{y}_{-1}^{*}=\left(y_{2}, \ldots, y_{n-m}\right)^{T}$ is the vector of the last $n-m-1$ elements of $\mathbf{y}^{*}$. After dividing the last $(n-m-1)$ equations of (A8) by its first equation we immediately get that

$$
\mathbf{y}^{*}=y_{1}^{*}\left(1, \mathbf{k}^{T}\right)^{T}
$$

where

$$
\mathbf{k}=\frac{Q_{2}^{-1} \Delta}{Q_{1}^{-1} \Delta}
$$

and $Q_{1}^{-1}$ and $Q_{2}^{-1}$ take the form (A7). Taking into account (7), (A3) and (A5), we simplify the first equation of (A8) for element $y_{1}^{*}$ as follows

$$
\begin{aligned}
& u_{1}\left(\boldsymbol{\pi}_{1}^{* T} Q \pi_{1}^{*}+2 \mathbf{d}_{2}^{T}\left(\Sigma_{21}-\Sigma_{22} D_{21}\right) \pi_{1}^{*}+\mathbf{d}_{2}^{T} \Sigma_{22} \mathbf{d}_{2}\right) y_{1}^{*} \\
& =u_{1}\left(f_{0}+y_{1}^{* 2}\left(1, \mathbf{k}^{T}\right) Q\left(1, \mathbf{k}^{T}\right)^{T}\right) y_{1}^{*} \\
& =\frac{1}{2} Q_{1}^{-1} \Delta u_{2}\left(\boldsymbol{\mu}_{2}^{T} \mathbf{d}_{2}+\boldsymbol{\Delta}^{T}\left(\mathbf{x}_{1}^{0}+y_{1}^{*}\left(1, \mathbf{k}^{T}\right)^{T}\right)\right) .
\end{aligned}
$$


In Landsman (2008a) (eq. 29) it was shown that

$$
\left(1, \mathbf{k}^{T}\right) Q\left(1, \mathbf{k}^{T}\right)^{T}=\frac{1}{\left(Q_{1}^{-1} \boldsymbol{\Delta}\right)^{2}} \Delta^{T} Q^{-1} \boldsymbol{\Delta}
$$

which we substitute into (A11) to obtain the one-dimensional equation

$$
u_{1}\left(f_{0}+y_{1}^{* 2} \frac{1}{\left(Q_{1}^{-1} \boldsymbol{\Delta}\right)^{2}} \Delta^{T} Q^{-1} \boldsymbol{\Delta}\right) \frac{y_{1}^{*}}{Q_{1}^{-1} \boldsymbol{\Delta}}=\frac{1}{2} u_{2}\left(\boldsymbol{\mu}_{2}^{T} \mathbf{d}_{2}+\boldsymbol{\Delta}^{T}\left(\mathbf{x}_{1}^{0}+y_{1}^{*}\left(1, \mathbf{k}^{T}\right)^{T}\right)\right) .
$$

From (A13) and from the positivity of $u_{1}$ and $u_{2}$ it follows that $w^{*}$,

$$
w^{*}=\frac{y_{1}^{*}}{Q_{1}^{-1} \Delta}>0,
$$

should satisfy the following equation

$$
u_{1}\left(f_{0}+b^{2} w^{2}\right) w=\frac{1}{2} u_{2}\left(\boldsymbol{\mu}_{2}^{T} \mathbf{d}_{2}+\boldsymbol{\Delta}^{T}\left(\mathbf{x}_{1}^{0}+w Q_{1}^{-1} \boldsymbol{\Delta}\left(1, \mathbf{k}^{T}\right)^{T}\right)\right) .
$$

Furthermore, notice that

$$
Q_{1}^{-1} \boldsymbol{\Delta}\left(1, \mathbf{k}^{T}\right)^{T}=Q_{1}^{-1} \boldsymbol{\Delta}\left(1, \frac{\left(Q_{\mathbf{2}}^{-1} \boldsymbol{\Delta}\right)^{T}}{Q_{1}^{-1} \boldsymbol{\Delta}}\right)^{T}=\left(Q_{1}^{-1} \boldsymbol{\Delta},\left(Q_{\mathbf{2}}^{-1} \boldsymbol{\Delta}\right)^{T}\right)^{T}=Q^{-1} \boldsymbol{\Delta},
$$

and taking into account (A5), Equation (A14) is straightforwardly reduced to Equation (10).

Suppose $w^{*}$ is the positive solution of (A14). Then from (A9) and (A15) follows that

$$
\mathbf{y}^{*}=w^{*} Q^{-1} \Delta .
$$

Now, since the ratio functional

$$
q(\pi)=\frac{p\left(\pi^{T} \boldsymbol{\mu}\right)}{v\left(\pi^{T} \Sigma \pi\right)}
$$

is the ratio of a convex or concave function and a convex function, our problem is in the field of fractional programming as described in Schaible and Ibaraki (1983). Therefore, since in the theory of fractional programming is known that a local maximum is global and unique, so does our solution of the maximization problem. Recall that $w^{*}$ is a unique positive solution of Equation (A14). Taking into account (A2), we finally conclude from (A16) that the maximizing vector of weights has the form

$$
\begin{aligned}
\pi^{*} & =\left(\left(\boldsymbol{\pi}_{1}^{0}+\mathbf{y}^{*}\right)^{T},\left(\mathbf{d}_{2}-D_{21} \pi_{1}^{0}-D_{21} \mathbf{y}^{*}\right)^{T}\right)^{T} \\
& =\pi^{0}+w^{*} \mathbf{z}
\end{aligned}
$$

where the vector $\mathbf{z}$ has the following partition

$$
\mathbf{z}=\left(\Delta^{T} Q^{-1},-\Delta^{T} Q^{-1} D_{12}\right)^{T} .
$$

Now let us return to the original maximization problem (11) subject to (5). We have shown that the unique solution of this problem can be written in the form (A17), where neither $\pi^{0}$ nor $\mathbf{z}$ depend on the functional $\mathcal{F}(\pi)$ but both depend only on the covariance matrix $\Sigma$, matrix $B$ and vectors $\mu$ and $\mathrm{c}$.

Consider the special case of functional $\mathcal{F}(\pi)$, when $t(x)=\ln x, p(x)=e^{x}$ and $v(x)=e^{\lambda x}, \lambda>0$. In this case, using (8) and (9), the functions $u_{1}$ and $u_{2}$ simply take the forms 


$$
u_{1}(x)=\lambda, u_{2}(x)=1
$$

and therefore, Equation (10) reduces to

$$
w^{*}=\frac{1}{2 \lambda}
$$

Note that this optimization problem takes the form

$$
\pi^{*}=\arg \inf _{B \pi=c}\left(-\mu^{T} \pi+\lambda\left(\pi^{T} \Sigma \pi\right)\right) .
$$

From the solution of the well known quadratic programming problem (see, for example, Luenberger (1984), chp. 14.1, eq. (9)) we can immediately conclude that

$$
\mathbf{z}=\Sigma^{-1} \boldsymbol{\mu}-\Sigma^{-1} B^{T}\left(B \Sigma^{-1} B^{T}\right)^{-1} B \Sigma^{-1} \mu .
$$

Proof of Corollary 1 . We show that the random vectors $\pi^{0 T} \mathbf{X}$ and $\mathbf{z}^{T} \mathbf{X}$ are orthogonal in the sense that

$$
\operatorname{cov}\left(\boldsymbol{\pi}^{0 T} \mathbf{X}, \mathbf{z}^{T} \mathbf{X}\right)=\pi^{0 T} \Sigma \mathbf{z}=0 .
$$

In fact,

$$
\begin{aligned}
\pi^{0 T} \Sigma \mathbf{z} & =\mathbf{c}^{T}\left(B \Sigma^{-1} B^{T}\right)^{-1} B \Sigma^{-1} \Sigma\left(\Sigma^{-1} \boldsymbol{\mu}-\Sigma^{-1} B^{T}\left(B \Sigma^{-1} B^{T}\right)^{-1} B \Sigma^{-1} \boldsymbol{\mu}\right) \\
& =\mathbf{c}^{T}\left(B \Sigma^{-1} B^{T}\right)^{-1} B \Sigma^{-1} \boldsymbol{\mu}-\mathbf{c}^{T}\left(B \Sigma^{-1} B^{T}\right)^{-1} B \Sigma^{-1} B^{T}\left(B \Sigma^{-1} B^{T}\right)^{-1} B \Sigma^{-1} \boldsymbol{\mu} \\
& =\mathbf{0}
\end{aligned}
$$

Then from (12) it follows that

$$
\left\{\begin{array}{l}
\mu^{*}=E\left(\boldsymbol{\pi}^{* T} \mathbf{X}\right)=\mu_{0}+w \mu_{\mathbf{z}} \\
\sigma^{* 2}=\operatorname{cov}\left(\boldsymbol{\pi}^{* T} \mathbf{X}\right)=\sigma_{0}^{2}+w^{2} \sigma_{\mathbf{z}}^{2}
\end{array},\right.
$$

where

$$
\begin{aligned}
& \mu_{0}=\boldsymbol{\mu}^{T} \boldsymbol{\pi}^{0}=\boldsymbol{\mu}^{T} \Sigma^{-1} B^{T}\left(B \Sigma^{-1} B^{T}\right)^{-1} \mathbf{c} \\
& \mu_{\mathbf{z}}=\boldsymbol{\mu}^{T} \mathbf{z}=\boldsymbol{\mu}^{T} \Sigma^{-1} \boldsymbol{\mu}-\left(B \Sigma^{-1} \boldsymbol{\mu}\right)^{T}\left(B \Sigma^{-1} B^{T}\right)^{-1}\left(B \Sigma^{-1} \boldsymbol{\mu}\right) \\
& \sigma_{0}^{2}=\pi^{0 T} \Sigma \boldsymbol{\pi}^{0}=\mathbf{c}^{T}\left(B \Sigma^{-1} B^{T}\right)^{-1} \mathbf{c}=f_{0} \\
& \sigma_{\mathbf{z}}^{2}=\mathbf{z}^{T} \Sigma \mathbf{z}=\boldsymbol{\mu}^{T} \Sigma^{-1} \boldsymbol{\mu}-\left(B \Sigma^{-1} \boldsymbol{\mu}\right)^{T}\left(B \Sigma^{-1} B^{T}\right)^{-1} B \Sigma^{-1} \boldsymbol{\mu}=\mu_{\mathbf{z}}
\end{aligned}
$$

Excluding $w$ from the system (A21) we obtain the parabolic $\left(\mu^{*}, \sigma^{* 2}\right)-$ efficient frontier

$$
\mu^{*}=\mu_{0}+\frac{\mu_{\mathbf{z}}}{\sigma_{\mathbf{z}}} \sqrt{\sigma^{* 2}-\sigma_{0}^{2}}=\mu_{0}+\sqrt{\mu_{\mathbf{z}}} \sqrt{\sigma^{* 2}-\sigma_{0}^{2}}, \sigma^{*} \geq \sigma_{\mathbf{z}}
$$

Now let us notice that any specific members of utility (3) would contain the risk parameter, say $\gamma$, that reflects to trade-off between expected return and risk, and there is one-to-one map between the set of possible meanings of this parameter, $\Gamma$, and subset of the positive part of real line, $R_{+}$. If some utility has no such parameter explicitly we say that $\Gamma$ contains only one value. For classical mean-variance portfolio $\gamma=\frac{1}{2 \lambda}$ and subset $\Gamma=R_{+}$. If $\Gamma \subset R_{+}$, the corresponding efficient portfolio belongs to that given for classical efficient portfolio. 


\section{References}

Bera, Samaresh, Praveen Gupta, and Sudip Misra. 2015. D2S: Dynamic demand scheduling in smart grid using optimal portfolio selection strategy. IEEE Transactions on Smart Grid 6: 1434-42.

Best, Michael J., and Robert R. Grauer. 1990. The efficient set mathematics when mean-variance problems are subject to general linear constraints. Journal of Economics and Business 42: 105-20.

Castellano, Rosella, and Roy Cerqueti. 2014. Mean-Variance portfolio selection in presence of infrequently traded stocks. European Journal of Operational Research 234: 442-49.

Elton, Edwin J.,and Martin J. Gruber. 1987. Modern Portfolio Theory and Investment Analysis. New York: Wiley.

Fletcher, Jonathan. 2015. Exploring the benefits of using stock characteristics in optimal portfolio strategies. The European Journal of Finance 23: 1-19.

Fulga, Cristinca. 2016. Portfolio optimization under loss aversion. European Journal of Operational Research 251: 310-22.

Graham, John R., Campbell R. Harvey, and Manju Puri. 2015. Capital allocation and delegation of decision-making authority within firms. Journal of Financial Economics 115: 449-70.

Landsman, Zinoviy, and Emiliano A. Valdez. 2003. Tail conditional expectations for elliptical distributions. North American Actuarial Journal 7: 55-71.

Landsman, Zinoviy. 2008a. Minimization of the root of a quadratic functional under an affine equality constraint. Journal of Computational and Applied Mathematics 216: 319-27.

Landsman, Zinoviy. 2008b. Minimization of the root of a quadratic functional under a system of affine equality constraints with application to portfolio management. Journal of Computational and Applied Mathematics 220: 739-48.

Landsman, Zinoviy, and Udi Makov. 2016. Minimization of a function of a quadratic functional with application to optimal portfolio selection. Journal of Optimization Theory and Application 170: 308-22.

Li, Bin, and Steven C. H. Hoi. 2014. Online portfolio selection: A survey. ACM Computing Surveys (CSUR) 46: 35. Luenberger, David G. 1984. Linear and Nonlinear Programming. Boston: Addison-Wesley.

Markowitz, Harry. 2014. Mean-variance approximations to expected utility. European Journal of Operational Research 234: 346-55.

Panjer, Harry H., and Phelim. P. Boyle, eds. 1998. Financial economics: With Applications to Investments, Insurance, and Pensions. Schaumburg: Society of Actuaries.

Qin, Zhongfeng. 2015. Mean-variance model for portfolio optimization problem in the simultaneous presence of random and uncertain returns. European Journal of Operational Research 245: 480-88.

Ray, Pritee, and Mamata Jenamani. 2016. Mean-variance analysis of sourcing decision under disruption risk. European Journal of Operational Research 250: 679-89.

Schaible, Siegfried, and Toshidide Ibaraki. 1983. Fractional programming. European Journal of Operational Research 12: $325-38$.

Sharpe, William F. 1966. Mutual fund performance. The Journal of Business 39: 119-38.

Sharpe, William F. 1998. The Sharpe Ratio. Streetwise-The Best of the Journal of Portfolio Management. Princeton: Princeton University Press, pp. 169-85.

Shen, Yang, Xin Zhang, and Tak Kuen Siu. 2014. Mean-variance portfolio selection under a constant elasticity of variance model. Operations Research Letters 42: 337-42.

Steinbach, Marc C. 2001. Markowitz Revisted: Mean-Variance models in financial portfolio analysis. SIAM Review 43: 31-85.

(C) 2018 by the authors. Licensee MDPI, Basel, Switzerland. This article is an open access article distributed under the terms and conditions of the Creative Commons Attribution (CC BY) license (http:/ / creativecommons.org/licenses/by/4.0/). 